Ciência Florestal, Santa Maria, v. 24, n. 2, p. 379-390, abr.-jun., 2014

ISSN 0103-9954

\title{
CARACTERIZAÇÃO FLORÍSTICA E ESTRUTURAL DE FRAGMENTOS DE MATAS DE GALERIA DA BACIA DO ALTO ARAGUAIA
}

\author{
FLORISTIC AND STRUCTURAL CHARACTERIZATION OF GALLERY FOREST FRAGMENTS \\ OF UPPER ARAGUAIA RIVER BASIN
}

\author{
Christian Dias Cabacinha ${ }^{1}$ Marco Aurélio Leite Fontes ${ }^{2}$
}

\begin{abstract}
RESUMO
As florestas da bacia do alto Araguaia estão diariamente expostas a vários agentes de degradação devido à intensa prática de agricultura nessa região. Vinte e dois fragmentos (de 10 a 169 ha) foram inventariados pelo método de quadrantes centrados, com o objetivo de caracterizar a estrutura da vegetação e criar um banco de dados de informações voltado para programas de restauração florestal na região. Foram amostradas 109 espécies pertencentes a 78 gêneros e 42 famílias. Dessas, 73,4\% apresentaram padrão de dispersão zoocórica e $69,7 \%$ foram classificadas como de estádios iniciais de sucessão (53,2\% secundárias iniciais e $16,5 \%$ pioneiras). O índice de Shannon foi 3,86 nats.ind $^{-1}$ e o de equabilidade de Pielou foi 0,82 . As estimativas da densidade e área basal, considerando todos os fragmentos, foram de 1.351 árvores.ha ${ }^{-1}$ e 19,28 m².ha ${ }^{-1}$, respectivamente. As áreas apresentaram uma menor riqueza (quando comparadas a matas de galerias mais conservadas), heterogeneidade nos índices de diversidade e equabilidade, tamanho reduzido das árvores e consequente baixa área basal, elevado número de espécies características de estádios intermediários de sucessão ecológica e colonização de espécies de cerrado e cerradão em áreas mais antropizadas, alterando a paisagem original. Tal situação, somada à importância dessas áreas para a conservação da biodiversidade e de serviços ecológicos (principalmente relativos à água), exige ações de proteção e manejo conservacionista que aproveitem o grande potencial regenerativo da área, dado pela existência de um grande número de espécies secundárias iniciais e predomínio das espécies zoocóricas.
\end{abstract}

Palavras-chave: mata de galeria; fitossociologia; conservação; cerrado brasileiro.

\section{ABSTRACT}

The forests of upper Araguaia river basin are daily exposed to degradation agents due to intense agriculture practices. Twenty two fragments (of 10 until 169 ha) were surveyed according to point-centered quarter method to characterize vegetation structure and to create a database to forest restoration. One hundred and nine (109) species, belonging to 78 genus and 42 families, were sampled where $73.4 \%$ revealed zoochorous dispersal pattern, and $69.7 \%$ were classified to initial sucessional category. Shannon index and Pielou equability index were 3.86 nats. ind ${ }^{-1}$ and 0.82 , respectively. Density and total basal area estimated were 1,351 trees.ha $\mathrm{a}^{-1}$ and $19.28 \mathrm{~m}^{2}$.ha $\mathrm{a}^{-1}$. The areas showed lower richness, Shannon and Pielou heterogeneity indices, lower basal area, and high number of species of intermediate stage of ecological sucession and colonization of cerrado and cerradão species in disturbed areas, altering the original landscape. Such situation, added to the importance of those areas for the biodiversity conservation and ecological services (mainly relative to the water), demands protection actions and management that use the great regenerative potential of the area, given by the existence of a great number of initial secondary species and the prevalence of zoochoric species.

Keywords: gallery forest; phyitosociology; conservation; Brazilian savanna.

1 Engenheiro Florestal, Dr., Professor Adjunto do Instituto de Ciências Agrárias, Universidade Federal de Minas Gerais, Av. Universitária, 1000, CEP 39404-547, Montes Claros (MG), Brasil. cabacinha@ufmg.br

2 Engenheiro Florestal, Dr., Professor Adjunto do Departamento de Ciências Florestais, Universidade Federal de Lavras, Campus Universitário, Caixa Postal 3037, CEP 37200-000, Lavras (MG), Brasil. fontes@dcf.ufla.br

Recebido para publicação em 27/01/2012 e aceito em 20/12/2012 


\section{INTRODUÇÃO}

Nos anos 70, com a criação do Programa de Desenvolvimento do Centro-Oeste, o POLOCENTRO, os agricultores foram atraídos para o Cerrado, pela grande disponibilidade de terras a preços mais baixos que as do sul do país e pelos incentivos fiscais para a abertura de novas áreas. A soja introduzida na região teve um crescimento considerável, em consequência da demanda crescente pelo produto nos mercados internacionais. Entretanto, esse modelo de ocupação trouxe sérios problemas de ordem ambiental, devido ao uso inadequado dos recursos naturais. Práticas como o correntão e queimadas, inclusive sucessivas, aliadas à produção de carvão vegetal, foram comuns na fase do desmatamento intensivo. Segundo Castro et al. (2004), tais práticas foram ainda agravadas pela falta de adoção ampla de práticas conservacionistas de uso e manejo das terras, principalmente para as áreas mais suscetíveis, que se somaram à falta ou insuficiência de fiscalização pelos órgãos competentes, além da orientação técnica, fragmentando e reduzindo drasticamente as diferentes fisionomias naturais do Cerrado.

De acordo com Felfili (1995; 2001a), das fitofisionomias do bioma Cerrado, as matas de galeria são as que possuem maior complexidade estrutural e maior biodiversidade proporcional à área que ocupam, ou seja, cerca de 5\% do bioma (MENDONÇA et al., 1998). Além disso, têm conhecida importância na proteção dos cursos d'água, na manutenção da fauna silvestre e ainda são corredores de biodiversidade (RESENDE, 1998). Atualmente, as formações florestais na bacia do alto rio Araguaia, em especial as matas de galeria, encontram-se bastante fragmentadas e isoladas, cercadas por agricultura (principalmente soja e algodão) e por pastos, além de expostas diariamente a vários agentes de degradação, o que pode limitar a colonização de espécies (TABARELLI et al., 2005). Esta região é carente de informações ecológicas, sobretudo em levantamentos florísticos e fitossociológicos. Segundo Silva Júnior (2004), no Brasil, a composição florística de comunidades vegetais ainda é pouco conhecida e, em razão do lamentável estado de conservação, estudos nessa área se tornaram de grande importância, sobretudo os que caracterizem a sucessão das comunidades e a síndrome de dispersão de sementes, que influencia a colonização de habitats e a manutenção da biodiversidade em paisagens fragmentadas.

O objetivo deste trabalho foi caracterizar a riqueza e a estrutura da vegetação de fragmentos de mata de galeria da bacia do alto rio Araguaia, bem como determinar o status de conservação da vegetação e subsidiar programas de restauração florestal na região.

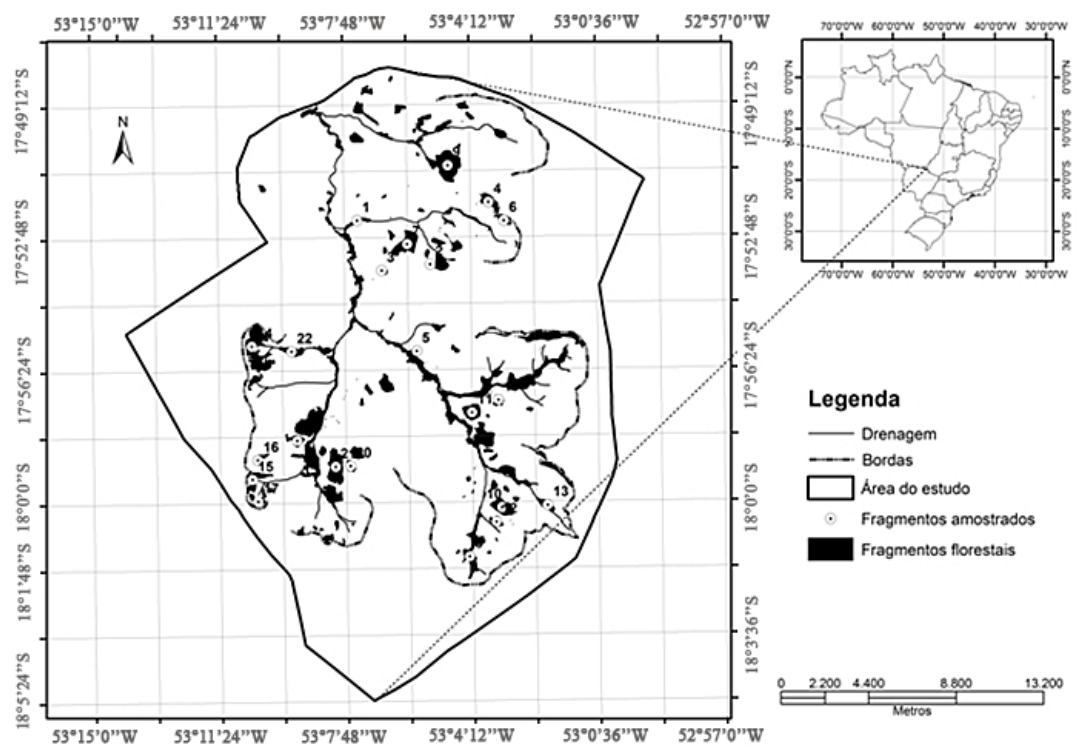

FIGURA 1: Localização da área e dos fragmentos estudados. Bacia do alto Araguaia, municípios de Mineiros, GO, e Alto Araguaia, MT, Brasil.

FIGURE 1: Area and fragments location. Upper Araguaia river basin. Mineiros, GO state and Alto Araguaia, MT state, Brazil. 


\section{MATERIAL E MÉTODOS}

\section{Localização e caracterização da área de estudo}

Aárea de estudo abrange o extremo sudoeste do estado de Goiás no município de Mineiros e o sul do estado do Mato Grosso no município de Alto Araguaia, bem próxima à divisa destes dois estados com o Mato Grosso do Sul. Insere-se no quadrante formado entre as coordenadas 17\%49'12'S./

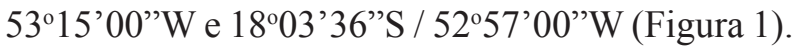

De acordo com a classificação climática de Köppen, a região apresenta clima do tipo Aw, caracterizado por ser tropical chuvoso, com verões quentes e invernos secos, cujas respectivas temperaturas médias são de $32^{\circ} \mathrm{C}$ e $18^{\circ} \mathrm{C}$, com média anual de $22^{\circ} \mathrm{C}$ (OLIVEIRA et al., 2003). A precipitação anual varia entre $1.500 \mathrm{~mm}$ e $1.650 \mathrm{~mm}$; o solo predominante na área de estudo é do tipo Neossolo Quartzarênico, sendo encontrado também nos fundos de vale Neossolos Quartzarênicos Hidromórficos e Gleissolos, e Latossolos VermelhoAmarelo nas cabeceiras de drenagem. A altitude média da região é de 800 metros (CASTRO et al., 2005).

A partir de uma classificação não supervisionada de cena TM/Landsat de junho de 2006 e da geração de um mapa do uso do solo da região estudada, verificou-se que, dos $52.214,70$ ha da área do estudo, 41.704,94 (79,87 \%) são áreas antropizadas com agricultura e pastagem. Para as fisionomias naturais utilizou-se a terminologia fitofisionômica proposta por Ribeiro e Walter (1998) e, após validação em campo, verificou-se que 6.466,68 (12,38\%) são de formações savânicas, $1.322,87(2,53 \%)$ são de formações campestres e $2.370,94(4,54 \%)$ são de formações florestais representadas por matas de galeria. A classificação ainda apresentou cerca de 349,27 hectares representados por corpos d'água.

\section{Amostragem e análise dos dados}

A área apresenta 198 fragmentos de floresta, dos quais 146 possuem menos que 10 ha e correspondem a $73 \%$. Dos 52 fragmentos com área superior a 10 ha, 22 foram amostrados aleatoriamente para o presente estudo (com tamanhos mínimo e máximo de 10 e 169 ha, respectivamente). Os fragmentos com menos de 10 ha foram excluídos por dois motivos básicos: os fragmentos com área inferior são fortemente influenciados pelos efeitos de borda (OLIVEIRA-FILHO et al., 1997); além disso, as imagens TM/Landsat permitem trabalhos em escala máxima ideal de 1:50.000, sendo qualquer fragmento de área inferior a 10 ha dificilmente visualizado nessa escala de trabalho.

Para caracterizar a estrutura e a composição florística de cada uma das áreas selecionadas, usou-se o método de quadrantes centrados (MUELLER-DOMBOIS e ELLENBERG 1974; MARTINS, 1991) que, conforme Moscovich et al. (1999), permite uma melhor cobertura espacial das áreas amostradas e, por consequência, uma maior representação e conhecimento da floresta em campo. Em cada área, lançaram-se 15 pontos quadrantes, onde foram amostrados os indivíduos lenhosos vivos com diâmetro a altura do peito (DAP, 1,3 m) maior ou igual a $5 \mathrm{~cm}$. Os indivíduos amostrados que não puderam ser identificados em campo foram coletados e posteriormente herborizados. Identificou-se o material botânico coletado com chaves de identificação baseadas em caracteres vegetativos, por comparação com exsicatas depositadas no Herbário BHCB da Universidade Federal de Minas Gerais e por consultas a especialistas. As espécies foram classificadas nas famílias reconhecidas pelo APGIII (2009).

Para verificação da suficiência amostral foi construída uma curva espécie-área e avaliada por regressão linear com resposta em platô (REGRELRP) do Sistema para Análises Estatísticas SAEG V.5.0 (GOMIDE, et al., 2006; ALVES JÚNIOR, et al., 2007), que, segundo Ferreira (1988), objetiva reduzir a subjetividade do método de determinação de área mínima, tradicionalmente feito pela curva espécie-área. $\mathrm{O}$ procedimento é apropriado para análise de regressão de modelos descontínuos, compostos de uma porção linear crescente e outra na forma de platô (SAEG, 1997) e, no gráfico determinado pelo procedimento, considera-se o número mínimo de pontos a serem amostrados aquele indicado pela intersecção entre as referidas porções. Para isso, considerou-se como unidade amostral o conjunto de 15 pontos lançados em cada fragmento.

Os descritores fitossociais foram calculados de acordo com Mueller-Dombois e Ellenberg (1974) e Castro (1987). Para toda a comunidade vegetal arbustivo-arbórea amostrada calculou-se a riqueza, densidade total e área basal (MUELLERDOMBOIS \& ELLENBERG 1974; MARTINS, 1991), bem como os índices de diversidade de Shannon e equabilidade de Pielou (MAGURRAN, 
1988). Toda análise foi realizada no programa Mata Nativa versão 2.08 (CIENTEC, 2007). A área foi comparada a outros levantamentos em matas de galeria no Brasil central.

Determinou-se a síndrome de dispersão dos frutos de cada espécie amostrada para estimar a oferta de frutos para a fauna da região. Para isso foram utilizadas informações sobre a biologia das espécies na literatura (OLIVEIRA \& MOREIRA 1992; MORELLATO \& LEITÃO-FILHO 1995; OLIVEIRA-FILHO et al., 1995; BARROSO et al., 1999; NUNES et al., 2003; TONIATO \& OLIVEIRA-FILHO, 2004) acrescidas das observações de campo dos autores.

Para a classificação sucessional, as espécies amostradas foram classificadas (a partir de pesquisa bibliográfica), como pioneiras, secundárias iniciais e secundárias tardias conforme critério de classificação sucessional sugeridos por Gandolfi et al. (1995). A vegetação foi classificada de acordo com seu estádio sucessional, adotando-se um dos critérios propostos por Budowski (1970): a proporção relativa entre o número de indivíduos de espécies iniciais (pioneiras e secundárias iniciais) e o de tardias (secundárias tardias e climácicas) que compõem o dossel da mata, considerando-se mais de $50 \%$ dos indivíduos de um estádio como determinante deste. Foram consideradas como espécies sem caracterização as espécies nativas para as quais não foram encontradas citações na literatura.

\section{RESULTADOS E DISCUSSÃO}

\section{Amostragem}

De acordo com os resultados do procedimento REGRELRP, pôde-se observar que a forma em platô é obtida na décima sétima unidade amostral, que corresponde a 255 pontos ou 900 árvores (Figura 2). Assim, pelo método pode-se considerar que a amostragem realizada para a área foi suficiente para caracterizar a comunidade arbórea da área em estudo. No entanto, o número de espécies parece continuar aumentando com o maior número de amostras, naquilo que Gomide et al. (2005) descrevem como um possível falso platô, não claro em sua estabilidade. Segundo Silva Júnior (2005), o ambiente de mata de galeria naturalmente impõe dificuldades à estabilização das curvas do tipo espécie-área devido à sua diversidade de condições de umidade e fertilidade dos solos, com consequente diversificação da vegetação e, ainda, o aumento contínuo do número de espécies indicaria comunidades sob distúrbios e, por isso, em fases inicias de sucessão, com pouca diferenciação de nichos, conforme Leps e Stursa (1989), o que corrobora com as observações na área de estudo, visto a amostragem ser similar àquelas encontradas em grande parte de estudos fitossociológicos.

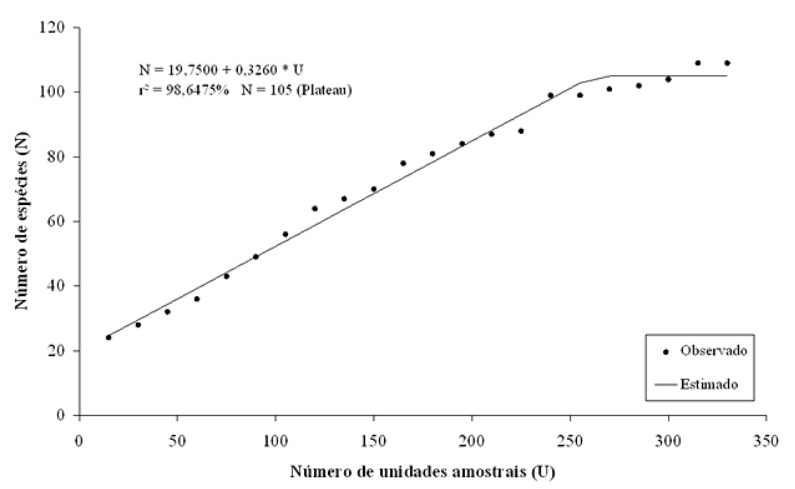

FIGURA 2: Representação gráfica da suficiência amostral. Número de unidades amostrais (U) versus número de espécies $(\mathrm{N})$. Bacia do alto Araguaia, municípios de Mineiros, GO, e Alto Araguaia, MT, Brasil.

FIGURE 2: Graphical representation of sampling sufficiency, Sampling units (U) versus number of species $(\mathrm{N})$. Upper Araguaia river basin. Mineiros, GO state and Alto Araguaia, MT state, Brazil.

\section{Diversidade florística}

A Tabela 1 mostra a riqueza e os índices de diversidade de Shannon e de equabilidade de Pielou para os 22 fragmentos amostrados e para a amostra total.

Os 1.320 indivíduos registrados distribuíram-se em 109 espécies em toda área amostrada. O índice de equabilidade de Pielou foi de 0,82 (o que indica pouca dominância de espécie, ou seja, que os indivíduos estão bem distribuídos entre as espécies) e o índice de diversidade de Shannon de 3,86 nats. ind ${ }^{-1}$, valores compatíveis para a faixa encontrada para as matas de galeria do Brasil central, conforme Silva Júnior et al. (1998, 2001), mesmo considerando-se apenas levantamentos que fizeram uso do método de pontos quadrantes (e.g. Silva Júnior, 2004 e 2005). O mesmo vale para os índices encontrados para cada fragmento deste estudo, ou 
TABELA 1: Localização, latitudes, longitudes, área (ha), riqueza florística (S), diversidade (H') e equabilidade (J) dos fragmentos amostrados. Bacia do alto Araguaia, municípios de Mineiros, GO e Alto Araguaia, MT, Brasil.

TABLE 1: Location, latitudes, longitudes, area (ha), tree number (N), floristic richness (S), maximum diversity (ln S), diversity (H') and equability (J) of the forest fragments sampled. Upper Araguaia river basin, Mineiros, GO state and Alto Araguaia, MT state, Brazil.

\begin{tabular}{|c|c|c|c|c|c|c|}
\hline Local & Latitudes & Longitudes & Área (ha) & $S$ & $\mathrm{H}^{\prime}$ & $\mathrm{J}$ \\
\hline 1 & $17^{\circ} 52^{\prime} 18,39^{\prime \prime}$ & $53^{\circ} 07^{\prime} 25,54^{\prime \prime}$ & 62,55 & 24 & 2,77 & 0,87 \\
\hline 2 & $17^{\circ} 53^{\prime} 32,20^{\prime \prime}$ & $53^{\circ} 05^{\prime} 23,76^{\prime \prime}$ & 10,18 & 13 & 2,10 & 0,82 \\
\hline 3 & $17053^{\prime} 37,73^{\prime \prime}$ & $53^{\circ} 06^{\prime} 44,95^{\prime \prime}$ & 14,12 & 13 & 2,09 & 0,82 \\
\hline 4 & $17^{\circ} 51^{\prime} 52,56 "$ & $53^{\circ} 03^{\prime} 42,28^{\prime \prime}$ & 37,67 & 14 & 2,22 & 0,84 \\
\hline 5 & $17^{\circ} 55^{\prime} 52,42^{\prime \prime}$ & $53^{\circ} 05^{\prime} 49,60^{\prime \prime}$ & 105,66 & 19 & 2,51 & 0,85 \\
\hline 6 & $17^{\circ} 52^{\prime} 22,08^{\prime \prime}$ & $53^{\circ} 03^{\prime} 14,61^{\prime \prime}$ & 18,16 & 20 & 2,29 & 0,76 \\
\hline 7 & $17^{\circ} 53^{\prime} 00,83^{\prime \prime}$ & $53^{\circ} 06^{\prime} 02,51^{\prime \prime}$ & 88,71 & 24 & 2,94 & 0,92 \\
\hline 8 & $18^{\circ} 01^{\prime} 30,08^{\prime \prime}$ & $53^{\circ} 04^{\prime} 19,19^{\prime \prime}$ & 107,14 & 24 & 2,80 & 0,88 \\
\hline 9 & $17^{\circ} 50^{\circ} 51,67^{\prime \prime}$ & $53^{\circ} 04^{\prime} 50,55^{\prime \prime}$ & 151,85 & 20 & 2,71 & 0,90 \\
\hline 10 & $18^{\circ} 00^{\prime} 10,74^{\prime \prime}$ & $53^{\circ} 03^{\prime} 20,14^{\prime \prime}$ & 62,17 & 19 & 2,65 & 0,90 \\
\hline 11 & $17^{\circ} 57^{\prime} 32,06^{\prime \prime}$ & $53^{\circ} 04^{\prime} 13,65^{\prime \prime}$ & 53,96 & 28 & 3,08 & 0,92 \\
\hline 12 & $18^{\circ} 00^{\prime} 34,72^{\prime \prime}$ & $53^{\circ} 03^{\prime} 33,06^{\prime \prime}$ & 25,38 & 21 & 2,78 & 0,91 \\
\hline 13 & $18^{\circ} 00^{\prime} 05,20^{\prime \prime}$ & $53^{\circ} 02^{\prime} 06,34^{\prime \prime}$ & 22,57 & 15 & 2,42 & 0,89 \\
\hline 14 & $17^{\circ} 55^{\prime} 43,20^{\prime \prime}$ & $53^{\circ} 10^{\prime} 30,05^{\prime \prime}$ & 169,10 & 19 & 2,30 & 0,78 \\
\hline 15 & $17^{\circ} 59^{\prime} 20,92 "$ & $53^{\circ} 10^{\prime} 30,05^{\prime \prime}$ & 46,13 & 15 & 2,34 & 0,86 \\
\hline 16 & $17^{\circ} 58^{\prime} 49,55^{\prime \prime}$ & $53^{\circ} 10^{\prime} 20,82^{\prime \prime}$ & 20,41 & 24 & 2,91 & 0,92 \\
\hline 17 & $17^{\circ} 59^{\prime} 55,98^{\prime \prime}$ & $53^{\circ} 10^{\prime} 18,98^{\prime \prime}$ & 33,83 & 11 & 2,00 & 0,83 \\
\hline 18 & $17^{\circ} 58^{\prime} 16,39^{\prime \prime}$ & $53^{\circ} 09^{\prime} 10,71^{\prime \prime}$ & 30,54 & 22 & 2,94 & 0,95 \\
\hline 19 & $17^{\circ} 57^{\prime} 13,61 "$ & $53^{\circ} 03^{\prime} 25,68^{\prime \prime}$ & 20,34 & 17 & 2,28 & 0,81 \\
\hline 20 & $17^{\circ} 59^{\prime} 00,62^{\prime \prime}$ & $53^{\circ} 07^{\prime} 38,46^{\prime \prime}$ & 20,31 & 15 & 2,48 & 0,92 \\
\hline 21 & $17^{\circ} 58^{\prime} 58,78^{\prime \prime}$ & $53^{\circ} 08^{\prime} 07,98^{\prime \prime}$ & 105,74 & 24 & 2,89 & 0,91 \\
\hline 22 & $17^{\circ} 55^{\prime} 50,58^{\prime \prime}$ & $53^{\circ} 09^{\prime} 21,78^{\prime \prime}$ & 38,36 & 19 & 2,68 & 0,91 \\
\hline Total & - & - & $1.244,87$ & 109 & 3,86 & 0,82 \\
\hline
\end{tabular}

seja, Shannon variando de 2,00 a 3,08 nats. ind $^{-1}$ e a equabilidade de 0,76 a 0,95 (não havendo relações entre os índices e as áreas dos fragmentos). Embora tal variação possa ser explicada pela natural diversidade de habitats nas matas de galeria (SILVA JÚNIOR, 2005), isto também ocorreu em consequência de fatores de degradação que foram observados em campo e que determinam mudanças consideráveis na diversidade dos fragmentos, como a presença de gado, cortes seletivos de madeira e invasão por gramíneas exóticas em quase todos, mas em graus variados de impacto.

\section{Estrutura fitossociológica}

Amostraram-se 1.320 indivíduos, sendo a área equivalente amostrada de 0,98 ha e a distância média entre árvores de $2,72 \mathrm{~m}$, que resultou na densidade total de 1.351 árvores.ha $^{-1}$ e na área basal total de $19,28 \mathrm{~m}^{2}$.ha-1. O DAP médio foi de $11,5 \pm 7,1 \mathrm{~cm}$ (máximo de 46,8 $\mathrm{cm}$ ) e a altura média $10,3 \pm 5,1 \mathrm{~m}$ (máxima de 30,0 m). Foram levantadas 109 espécies pertencentes a 78 gêneros e 42 famílias (Tabela 2).

Marimon et al. (2002) analisaram a florística e a fitossociologia de uma mata de galeria em uma unidade de conservação em Nova Xavantina, MT, e encontraram 129 espécies pertencentes a 105 gêneros e 47 famílias, sendo a densidade total de 1.023 árvores.ha ${ }^{-1} \mathrm{e}$ a área basal estimada de 20,44 $\mathrm{m}^{2} \cdot \mathrm{ha}^{-1}$. Comparativamente à área do presente estudo, a densidade menor e a área basal maior indicam a existência de árvores de maior porte e, somada ao maior número de espécies, uma área em estádio de maturidade mais avançado. 
TABELA 2: Parâmetros fitossociológicos, síndrome de dispersão (SD) e categorias de sucessão (CS) das espécies registradas em levantamento fitossociológico em matas de galeria na bacia do alto Araguaia, municípios de Mineiros, GO e Alto Araguaia, MT, Brasil.

TABLE2: Phytosociological parameters, dispersal syndromes(SD) and successional category (CS) of trees sampled. Upper Araguaia river basin, Mineiros, GO state and Alto Araguaia, MT state, Brazil.

\begin{tabular}{|c|c|c|c|c|c|c|}
\hline Espécies & $\mathrm{DR}$ & DoR & FR & VI & SD & CS \\
\hline Bocageopsis mattogrossensis (R.E.Fr.) R.E.Fr. & 13,9 & 9,9 & 10,9 & 34,6 & Zoo & SI \\
\hline Sclerolobium paniculatum Vog. & 5,9 & 10,6 & 4,3 & 20,8 & An & SI \\
\hline Tapirira guianensis Aubl. & 3,9 & 5,6 & 4,3 & 13,8 & Zoo & $\mathrm{Pi}$ \\
\hline Nectandra warmingii Meisn. & 3,6 & 4,3 & 3,6 & 11,5 & Zoo & ST \\
\hline Licania kunthiana Hook.f. & 4,0 & 2,4 & 3,8 & 10,2 & Zoo & SI \\
\hline Myrcia splendens (Sw.) DC. & 3,5 & 2,7 & 3,7 & 9,9 & Zoo & $\mathrm{SC}$ \\
\hline Ocotea aciphylla (Nees) Mez & 3,0 & 3,4 & 3,2 & 9,5 & Zoo & SI \\
\hline Matayba guianensis Aubl. & 2,1 & 5,0 & 2,4 & 9,5 & Zoo & SI \\
\hline Copaifera langsdorffii Desf. & 2,4 & 4,1 & 2,7 & 9,2 & Zoo & ST \\
\hline Miconia chartacea Triana & 3,6 & 1,4 & 4,0 & 9,0 & Zoo & ST \\
\hline Maprounea guianensis Aubl. & 2,4 & 1,6 & 2,7 & 6,8 & Zoo & SI \\
\hline Sloanea guianensis (Aubl.) Benth. & 2,7 & 1,6 & 2,3 & 6,5 & Zoo & SI \\
\hline Inga ingoides (Rich.) Willd. & 2,3 & 1,6 & 2,4 & 6,4 & Zoo & $\mathrm{SC}$ \\
\hline Qualea multiflora Mart. & 2,2 & 1,7 & 2,2 & 6,2 & An & SI \\
\hline Xylopia aromatica (Lam.) Mart. & 2,3 & 1,1 & 2,5 & 5,9 & Zoo & $\mathrm{Pi}$ \\
\hline Emmotum nitens (Benth.) Miers & 1,7 & 2,4 & 1,9 & 5,9 & Zoo & SI \\
\hline Virola sebifera Aubl. & 2,3 & 0,9 & 2,4 & 5,7 & Zoo & $\mathrm{Pi}$ \\
\hline Eugenia lambertiana DC. & 1,7 & 2,2 & 1,5 & 5,3 & Zoo & SI \\
\hline Simarouba amara Aubl. & 1,9 & 1,0 & 2,2 & 5,2 & Zoo & SI \\
\hline Buchenavia capitata (Vahl.) Eichl. & 1,0 & 3,2 & 0,9 & 5,2 & Zoo & SI \\
\hline Matayba elaeagnoides Radlk. & 1,5 & 2,0 & 1,4 & 4,9 & Zoo & SI \\
\hline Guatteria nigrescens Mart. & 1,3 & 1,9 & 1,4 & 4,6 & Zoo & ST \\
\hline Machaerium acutifolium Vogel & 0,8 & 2,4 & 0,8 & 4,0 & An & Pi \\
\hline Duguetia marcgraviana Mart. & 1,2 & 1,6 & 1,0 & 3,9 & Zoo & $\mathrm{SC}$ \\
\hline Anadenanthera colubrina (Vell.) Brenan & 1,1 & 1,5 & 1,2 & 3,8 & An & SI \\
\hline Erythroxylum citrifolium A.St.-Hil. & 1,1 & 1,4 & 1,2 & 3,7 & Zoo & ST \\
\hline Nectandra cissiflora Nees & 1,4 & 1,0 & 1,3 & 3,6 & Zoo & ST \\
\hline Quiina rhytidopus Tul. & 1,1 & 1,1 & 1,1 & 3,3 & Zoo & SI \\
\hline Guatteria sellowiana Schltdl. & 1,2 & 0,9 & 1,1 & 3,3 & Zoo & SI \\
\hline Roupala brasiliensis Klotz. & 1,3 & 0,5 & 1,5 & 3,2 & An & ST \\
\hline Ocotea corymbosa (Meisn.) Mez & 0,9 & 1,3 & 0,8 & 3,1 & Zoo & SI \\
\hline Amaioua guianensis Aubl. & 1,0 & 1,0 & 0,9 & 3,0 & Zoo & ST \\
\hline Sweetia fruticosa Spreng. & 0,9 & 0,9 & 1,0 & 2,9 & An & $\mathrm{Pi}$ \\
\hline Unonopsis lindmanii R.E.Fr. & 0,8 & 1,0 & 0,9 & 2,8 & Zoo & ST \\
\hline Mabea pohliana (Benth.) Müll.Arg. & 0,9 & 0,4 & 1,1 & 2,5 & Zoo & $\mathrm{SC}$ \\
\hline Buchenavia tomentosa Eichler & 0,5 & 1,0 & 0,6 & 2,1 & Zoo & $\mathrm{Pi}$ \\
\hline Eriotheca candolleana (K.Schum.) A.Robyns & 0,8 & 0,5 & 0,8 & 2,1 & An & SI \\
\hline Myrcia rufipes DC. & 0,8 & 0,3 & 0,8 & 1,9 & Zoo & ST \\
\hline Inga heterophylla Willd. & 0,5 & 0,7 & 0,6 & 1,8 & Zoo & SI \\
\hline Cecropia pachystachya Trécul & 0,6 & 0,5 & 0,7 & 1,8 & Zoo & $\mathrm{Pi}$ \\
\hline
\end{tabular}

Continua $\ldots$ 
TABELA 2: Continuação ...

TABLE 2: Continued ...

\begin{tabular}{|c|c|c|c|c|c|c|}
\hline Espécies & DR & DoR & FR & VI & SD & $\mathrm{CS}$ \\
\hline Kielmeyera coriacea Mart. \& Zucc. var.coriacea & 0,6 & 0,4 & 0,7 & 1,8 & An & SI \\
\hline Guarea guidonia (L.) Sleumer & 0,6 & 0,5 & 0,6 & 1,6 & Zoo & SI \\
\hline Hirtella glandulosa Spreng. & 0,4 & 0,8 & 0,4 & 1,6 & Zoo & SI \\
\hline Hirtella gracilipes (Hook.f.) Prance & 0,5 & 0,5 & 0,5 & 1,4 & Zoo & SI \\
\hline Pouteria gardneri (Mart. \& Miq.) Baehni & 0,5 & 0,1 & 0,7 & 1,3 & Zoo & ST \\
\hline Tocoyena brasiliensis Mart. & 0,5 & 0,4 & 0,5 & 1,3 & Zoo & ST \\
\hline Pseudolmedia laevigata Trécul & 0,2 & 0,8 & 0,3 & 1,3 & Zoo & SI \\
\hline Ouratea spectabilis (Mart. \& Engl.) Engl. & 0,5 & 0,1 & 0,6 & 1,2 & Zoo & $\mathrm{SC}$ \\
\hline Erythroxylum daphnites Mart. & 0,5 & 0,3 & 0,5 & 1,2 & Zoo & $\mathrm{SC}$ \\
\hline Myrcia linguaeformis Kiaersk. & 0,5 & 0,3 & 0,5 & 1,2 & Zoo & $\mathrm{SC}$ \\
\hline Myrsine guianensis (Aubl.) Kuntze & 0,4 & 0,3 & 0,5 & 1,1 & Zoo & $\mathrm{Pi}$ \\
\hline Ocotea spixiana (Nees) Mez & 0,4 & 0,4 & 0,3 & 1,1 & Zoo & SI \\
\hline Casearia gossypiosperma Briq. & 0,4 & 0,2 & 0,4 & 0,9 & Zoo & SI \\
\hline Pouteria ramiflora (Mart.) Radlk. & 0,4 & 0,2 & 0,3 & 0,9 & Zoo & ST \\
\hline Byrsonima intermedia A.Juss. & 0,4 & 0,2 & 0,4 & 0,9 & Zoo & SI \\
\hline Himatanthus drasticus (Mart.) Plumel & 0,3 & 0,2 & 0,4 & 0,9 & An & $\mathrm{Pi}$ \\
\hline Myrsine umbellata Mart. & 0,2 & 0,4 & 0,3 & 0,9 & Zoo & SI \\
\hline Luehea grandiflora Mart. \& Zucc. & 0,3 & 0,3 & 0,3 & 0,9 & An & $\mathrm{Pi}$ \\
\hline Qualea cordata (Mart.) Spreng. & 0,3 & 0,3 & 0,3 & 0,8 & An & $\mathrm{SC}$ \\
\hline Gomidesia lindeniana O.Berg. & 0,3 & 0,1 & 0,4 & 0,8 & Zoo & SI \\
\hline Talisia esculenta (A.St.-Hil.) Radlk. & 0,2 & 0,4 & 0,2 & 0,7 & Zoo & $\mathrm{Pi}$ \\
\hline Myrciaria floribunda (H.West ex Willd.) O.Berg & 0,2 & 0,4 & 0,2 & 0,7 & Zoo & SI \\
\hline $\begin{array}{l}\text { Licania octandra (Hoffmanns. ex Roem. \& Schult.) } \\
\text { Kuntze }\end{array}$ & 0,2 & 0,1 & 0,3 & 0,6 & Zoo & SI \\
\hline Cheiloclinium cognatum (Miers.) A.C.Sm. & 0,1 & 0,4 & 0,1 & 0,6 & Zoo & SI \\
\hline Guapira venosa (Choisy) Lundell & 0,2 & 0,1 & 0,3 & 0,6 & Zoo & SI \\
\hline Connarus suberosus Planch. var suberosus & 0,2 & 0,1 & 0,3 & 0,6 & Zoo & $\mathrm{SC}$ \\
\hline Licania apetala (E.Mey.) Fritsch & 0,2 & 0,1 & 0,3 & 0,6 & Zoo & SI \\
\hline Hymenaea courbaril L. & 0,2 & 0,1 & 0,3 & 0,6 & Zoo & ST \\
\hline Miconia cuspidata Mart. ex Naudin & 0,2 & 0,2 & 0,2 & 0,5 & Zoo & ST \\
\hline Xylopia sericea A.St.-Hil. & 0,2 & 0,2 & 0,2 & 0,5 & Zoo & SI \\
\hline Caryocar brasiliense Cambess. & 0,2 & 0,1 & 0,2 & 0,5 & Zoo & $\mathrm{Pi}$ \\
\hline Toulicia laevigata Radlk. & 0,2 & 0,1 & 0,2 & 0,5 & An & $\mathrm{Pi}$ \\
\hline Ocotea glaziovii Mez & 0,2 & 0,1 & 0,2 & 0,5 & Zoo & ST \\
\hline Cordia sellowiana Cham. & 0,2 & 0,1 & 0,2 & 0,5 & Zoo & SI \\
\hline Diptychandra aurantiaca Tul. & 0,1 & 0,3 & 0,1 & 0,5 & An & SI \\
\hline Nectandra cuspidata Nees & 0,2 & 0,1 & 0,2 & 0,5 & Zoo & SI \\
\hline Acosmium subelegans (Mohlenbr.) Yakovlev & 0,1 & 0,3 & 0,1 & 0,4 & An & $\mathrm{Pi}$ \\
\hline Guarea macrophylla Vahl & 0,2 & 0,2 & 0,1 & 0,4 & Zoo & SI \\
\hline Tabebuia aurea (Manso) Benth. \& Hook.f. ex S.Moore & 0,2 & 0,1 & 0,2 & 0,4 & An & $\mathrm{Pi}$ \\
\hline Plathymenia reticulata Benth. & 0,2 & 0,1 & 0,2 & 0,4 & An & SI \\
\hline Stryphnodendron obovatum Benth. & 0,2 & 0,1 & 0,2 & 0,4 & $\mathrm{Au}$ & $\mathrm{Pi}$ \\
\hline Bauhinia longifolia (Bong.) D.Dietr. & 0,2 & 0,1 & 0,2 & 0,4 & An & SI \\
\hline
\end{tabular}

Continua ... 
TABELA 2: Continuação ...

TABLE 2: Continued ...

\begin{tabular}{|c|c|c|c|c|c|c|}
\hline Espécies & DR & DoR & FR & VI & SD & $\mathrm{CS}$ \\
\hline Siparuna guianensis Aubl. & 0,2 & 0,0 & 0,2 & 0,4 & Zoo & SI \\
\hline Cupania vernalis Cambess. & 0,2 & 0,0 & 0,2 & 0,4 & Zoo & SI \\
\hline Prunus myrtifolia (L.) Urb. & 0,2 & 0,0 & 0,2 & 0,4 & Zoo & SI \\
\hline Combretum discolor Taub. & 0,1 & 0,2 & 0,1 & 0,3 & An & $\mathrm{SC}$ \\
\hline Aspidosperma discolor A.DC. & 0,2 & 0,1 & 0,1 & 0,3 & An & SI \\
\hline Annona cornifolia A.St.-Hil. & 0,1 & 0,1 & 0,1 & 0,3 & Zoo & SI \\
\hline Aspidosperma subincanum Mart. ex A.DC. & 0,1 & 0,1 & 0,1 & 0,3 & An & SI \\
\hline Diospyros sericea A.DC. & 0,2 & 0,0 & 0,1 & 0,3 & Zoo & SI \\
\hline Aspidosperma parvifolium A.DC. & 0,1 & 0,1 & 0,1 & 0,3 & An & ST \\
\hline Rudgea viburnoides (Cham.) Benth. & 0,1 & 0,1 & 0,1 & 0,2 & Zoo & SI \\
\hline Aspidosperma spruceanum Benth. ex Müll.Arg. & 0,1 & 0,0 & 0,1 & 0,2 & An & ST \\
\hline Couepia grandiflora (Mart. \& Zucc.) Benth. ex Hook.f. & 0,1 & 0,0 & 0,1 & 0,2 & Zoo & $\mathrm{Pi}$ \\
\hline Pterodon pubescens (Benth) Benth. & 0,1 & 0,0 & 0,1 & 0,2 & An & SI \\
\hline Astronium fraxinifolium Schott ex Spreng. & 0,1 & 0,0 & 0,1 & 0,2 & An & ST \\
\hline Byrsonima coriacea (Sw.) DC. & 0,1 & 0,0 & 0,1 & 0,2 & Zoo & SI \\
\hline Agonandra brasiliensis Miers ex Benth. \& Hook. & 0,1 & 0,0 & 0,1 & 0,2 & Zoo & ST \\
\hline Aiouea trinervis Meisn. & 0,1 & 0,0 & 0,1 & 0,2 & Zoo & ST \\
\hline Myrcia tomentosa (Aubl.) DC. & 0,1 & 0,0 & 0,1 & 0,2 & Zoo & SI \\
\hline Eriotheca gracilipes (K.Schum.) A.Robyns & 0,1 & 0,0 & 0,1 & 0,2 & An & SI \\
\hline Astronium nelsonrosae Santin & 0,1 & 0,0 & 0,1 & 0,2 & An & ST \\
\hline Myrsine parvula (Mez) Otegui & 0,1 & 0,0 & 0,1 & 0,2 & Zoo & $\mathrm{SC}$ \\
\hline Myrcia fallax (Rich.) DC. & 0,1 & 0,0 & 0,1 & 0,2 & Zoo & SI \\
\hline Casearia decandra Jacq. & 0,1 & 0,0 & 0,1 & 0,2 & Zoo & SI \\
\hline Dimorphandra mollis Benth. & 0,1 & 0,0 & 0,1 & 0,2 & $\mathrm{Au}$ & $\mathrm{Pi}$ \\
\hline Bowdichia virgilioides Kunth & 0,1 & 0,0 & 0,1 & 0,2 & An & SI \\
\hline Heisteria ovata Benth. & 0,1 & 0,0 & 0,1 & 0,2 & Zoo & SI \\
\hline Rhamnidium elaeocarpum Reissek & 0,1 & 0,0 & 0,1 & 0,2 & Zoo & SI \\
\hline Total geral & 100 & 100 & 100 & 300 & & \\
\hline
\end{tabular}

Em que: DR = densidade relativa (\%); DoR = dominância relativa (\%); FR = frequência relativa (\%); VI = valor de importância; $\mathrm{PI}$ = pioneira; $\mathrm{SI}$ = secundária inicial; $\mathrm{ST}$ = secundária tardia; $\mathrm{Zoo}=$ zoocórica; $\mathrm{An}$ = anemocórica; $\mathrm{Au}=$ autocórica; $\mathrm{SC}=$ sem classificação.

Silva Júnior (2004) e Silva Júnior (2005), em dois estudos em matas de galeria na Reserva Ecológica do IBGE, em Brasília, DF, cuja área encontra-se protegida de distúrbios, incluindo incêndios, há pelo menos 20 anos, encontrou riquezas semelhantes à deste estudo, porém, estruturas florestais mais avançadas. No primeiro estudo encontrou 110 espécies pertencentes a 91 gêneros e uma distância média entre árvores de $2,51 \mathrm{~m}$, que resultou na densidade total de 1.573 árvores. ha $^{-1} \mathrm{e}$ na área basal total de $38,5 \mathrm{~m}^{2}$.ha-1, valor este muito superior ao encontrado neste estudo e, no segundo trabalho, encontrou 99 espécies e 88 gêneros, sendo a distância média entre árvores de 2,28 m, o que resultou na densidade total de 1.971 árvores. ha ${ }^{-1} \mathrm{e}$ na área basal total de $38,8 \mathrm{~m}^{2}$. ha-1.

No estudo realizado por Dietzsch et al. (2006), a área basal estimada para a mata de galeria, considerando dois fragmentos estudados juntos, foi de $28,4 \mathrm{~m}^{2}$. ha' ${ }^{-1}$, também superior ao encontrado neste estudo, enquanto a densidade foi de 1.448 ind. ha ${ }^{-1}$. Estes valores são considerados dentro do intervalo de variação para as áreas basais e densidades médias das matas de galeria amostradas na região do Distrito Federal (BELTRÃO, 2003; FELFILI, 1995; SILVA JÚNIOR, 1995). Além 
disso, encontraram 79 espécies, sendo o menor valor devido a áreas inundáveis amostradas, naturalmente menos ricas, não sendo esse o caso no presente estudo, mas possivelmente também devido às perturbações antrópica existentes na área, principalmente de entorno, destacando-se erosões, lixo e fogo, dentre outros.

Teixeira e Rodrigues (2006) analisaram a florística e estrutura de uma mata de galeria no nordeste do estado de São Paulo, cujas margens encontravam-se sob o impacto de processos erosivos causados pelo pisoteio de gado. Encontraram 68 espécies pertencentes a 37 famílias; uma densidade de 1.774 ind. ha $^{-1}$ e uma área basal de $27,8 \mathrm{~m}^{2}$. ha-1 . Os dados sugerem que a pressão antrópica influencia na redução da riqueza, mas, ainda assim, a área do presente estudo mostra uma densidade e área basal inferiores. Embora a área sofra impactos antrópicos, comuns em ambientes cercados por matriz agropecuária no país, já citados, esses baixos valores se devem, notadamente, ao corte seletivo de lenha, cujos indícios foram encontrados em campo com alta frequência e a condições ambientais, sobretudo edáficas, resultando em baixos valores de diâmetros.

As 15 espécies com maior VI (Tabela 2) foram, em ordem decrescente, Bocageopsis mattogrossensis, Sclerolobium paniculatum, Tapirira guianensis, Nectandra warmingii, Licania kunthiana, Myrcia splendens, Ocotea aciphylla, Matayba guianensis, Copaifera langsdorffi, Miconia chartacea, Maprounea guianensis, Sloanea guianensis, Inga ingoides, Qualea multiflora e Xylopia aromática, que juntas corresponderam a $57 \%$ do total de VI, sendo todas espécies típicas de matas de galeria do cerrado (SANO et al., 2008; SILVA JÚNIOR et al., 1998; FELFILI et al., 2001b).

No levantamento, ainda foram observadas espécies típicas de cerrado sentido restrito: Connarus suberosus (3 indivíduos), Myrcia linguaeformis (6); e de cerrado e cerradão: Annona cornifolia (1), Caryocar brasiliensis (2), Combretum discolor (1), Himatanthus drasticus (4), Machaerium acutifolium (10), Ouratea spectabilis (7) e Stryphnodendron obovatum (2). Observou-se em campo que essas espécies colonizaram áreas de mata de galeria com alto grau de antropização, ou seja, comportando-se de modo oportunista e alterando a paisagem original. Além disso, podem ser consideradas de florestas estacionais semidecíduas do sudeste as espécies encontradas Guapira venosa (e.g. VALE et al., 2009; DIAS NETO et al., 2009) e Toulicia laevigata (e.g. OLIVEIRA-FILHO et al., 2004; BORÉM \& OLIVEIRA-FILHO, 2002).

\section{Síndromes de dispersão e classificação sucessional}

A classificação ecológica mostrou que, das 109 espécies encontradas, 20,2\% pertencem à categoria das secundárias tardias, 53,2\% às secundárias iniciais, $16,5 \%$ às pioneiras e 10,1\% não foram classificadas (Tabela 2). Com base no resultado pode-se dizer que os fragmentos amostrados encontram-se em estádio intermediário de sucessão e, de acordo com Oliveira-Filho (1989), se mantidos sob proteção podem retornar às condições anteriores, semelhantes à vegetação original, necessitando todos de ação urgente de fiscalização e cercamento. Entretanto, outros pontos devem ser considerados para garantir a restauração de uma comunidade florestal perturbada, além de sua proteção. É de suma importância a existência de fontes de propágulos próximas e a diminuição do grau de isolamento destas áreas, promovendo a diversificação genética das populações para garantia de sua viabilidade. A existência de um grande número de espécies secundárias iniciais indica que a área possui um grande potencial regenerativo, o que deve ser aproveitado durante ações de recomposição florestal desses fragmentos.

Dentre as espécies estudadas observou-se também o predomínio das espécies zoocóricas $(73,4 \%)$, seguidas por espécies anemocóricas $(24,8 \%)$ e autocóricas $(1,8 \%)$ (Tabela 2). Estes resultados corroboram com estudos realizados em diversas florestas. De acordo com Howe (1990), Morellato e Leitão-Filho (1992), Pinã-Rodrigues e Aguiar (1993) e Morellato (1995), a zoocoria tem sido a principal forma de dispersão de sementes de espécies arbóreas e arbustivas na maioria das florestas tropicais. Como exemplo da importância da zoocoria na sucessão florestal, Souza et al. (2007), quando estudaram a composição florística e a estrutura da vegetação arbustivo-arbórea no sub-bosque de um povoamento de Eucalyptus grandis W. Hill ex Maidem no município de ViçosaMG, verificaram que, das 50 espécies amostradas, $74 \%$ eram zoocóricas e 26\% anemocóricas.

Oliveira-Filho (1989) explicou o predomínio de espécies zoocóricas em matas de galeria afirmando que a distribuição de sementes em vegetações em forma de mosaico, geralmente se dá de forma zoocórica, pois os outros métodos de 
dispersão dependem da participação de água e vento que, associados à estrutura física desse ambiente, influenciam na dificuldade das sementes serem retiradas do mesmo, ao passo que o hábito alimentar dos agentes zoocóricos facilita a retirada. Nesse sentido, a necessidade de proteção das florestas de galeria devem se estender claramente também à sua fauna, como condição indispensável para a recuperação e conservação das áreas.

\section{CONCLUSÃO}

As matas de galerias da bacia do alto Araguaia, nos municípios de Mineiros (estado de Goiás) e Alto Araguaia (Mato Grosso), Brasil, apresentam uma menor riqueza (quando comparadas às mais conservadas), heterogeneidade nos índices de diversidade e equabilidade, tamanho reduzido das árvores e consequente baixa área basal, elevado número de espécies características de estádios intermediários de sucessão ecológica, e colonização de espécies de cerrado e cerradão nas áreas mais antropizadas, alterando a paisagem original. Dada a importância dessas áreas para a conservação da biodiversidade e de serviços ambientais, principalmente relativos à água, sugerem-se ações de proteção e manejo conservacionista que aproveitem o potencial regenerativo da área, dado pela existência de um grande número de espécies secundárias iniciais e predomínio de espécies zoocóricas.

\section{REFERÊNCIAS BIBLIOGRÁFICAS}

ALVES JÚNIOR, F. T. et al. Estrutura diamétrica e hipsométrica do componente arbóreo de um fragmento de mata atlântica, Recife-PE. Cerne, Lavras, v. 13, n. 1, p. 83-95, 2007.

ANGIOSPERM PHYLOGENY GROUP (APG). An Update of the Angiosperm Phylogeny Group classification for the orders and families of flowering plants: APG III. Botanical Journal of the Linnean Society, v. 161, p. 105-121, 2009.

BARROSO, G. M. et al. Frutos e sementes: morfologia aplicada à sistemática de dicotiledôneas. Viçosa: Ed. UFV, 1999.

BELTRÃO, L. Avaliação do efeito do entorno na integridade ecológica de uma mata de galeria inundável no Distrito Federal. 2003. 135 f. Dissertação (Mestrado em Ciências Florestais) - Universidade de Brasília, Brasília, 2003.

BORÉM, R. A. T.; OLIVEIRA-FILHO, A.
T. Fitossociologia do estrato arbóreo em uma topossequência alterada de mata atlântica, no municipio de Silva Jardim- RJ, Brasil. Revista Árvore, v. 26, n. 6, p. 727-742, 2002.

BUDOWSKI, G. The distinction between old secondary and climax species in tropical central american lowland rainforest. Tropical Ecology, Varanas, v. 11, p. 44-48, 1970.

CASTRO, A. A. J. F. Florística e fitossociologia de um cerrado marginal brasileiro, Parque Estadual de Vassununga, Santa Rita do Passa-Quatro, SP. 1987. 262 f. Dissertação (Mestrado em Biologia Vegetal) - Universidade Estadual de Campinas, Campinas, 1987.

CASTRO, S. S. de. Erosão hídrica na alta bacia do rio Araguaia: distribuição, condicionantes, origem e dinâmica atual. Revista do Departamento de Geografia, São Paulo. v. 17, p. 38-60, 2005.

CASTRO, S. S. de; XAVIER, L. S de.; BARBALHO, M. G. S. da. (Org.). Atlas geoambiental das nascentes dos rios Araguaia e Araguainha: condicionantes dos processos erosivos lineares. Goiânia: SEMARH, 2004. 74p

DIAS NETO, O. C. et al. Estrutura fitossociológica e grupos ecológicos em fragmento de floresta estacional semidecidual, Uberaba, Minas Gerais, Brasil. Rodriguésia, v. 60, n. 4, p. 1087-1100, 2009. DIETZSCH, L. et al. Caracterização da flora arbórea de dois fragmentos de mata de galeria do parque Canjerana, DF. Cerne, Lavras, v. 12, n. 3, p. 201-210, 2006.

EMBRAPA. Sistema brasileiro de classificação de solos. BrasíliaRio de Janeiro: Embrapa Solos, 1999. $412 \mathrm{p}$.

FELFILI, J. M. Diversity, structure and dynamics of a gallery forest in central Brazil. Vegetatio, v. 117, p. 1-15, 1995.

FELFILI, J. M. et al. O projeto biogeografia do bioma cerrado: hipóteses e padronização da metodologia. In: GARAY, I.; DIAS, B. Conservação da biodiversidade em ecossistemas tropicais. Petrópolis: [s.n.], 2001a. p.157-173.

FELFILI, J. M. et al. Flora fanerogâmica das matas de galeria e ciliares do Brasil Central. In: RIBEIRO, J. F.; FONSECA, C. E. L.; SOUZA-SILVA, J. C. (Ed.) Cerrado - caracterização e recuperação de matas de galeria. Planaltina: EMBRAPA-CPAC, 2001b. p.195-209.

FERREIRA, R. L. C. Análise estrutural da vegetaçãodaEstação Florestalde Experimentação de Açu, RN, como subsídio básico para o manejo florestal. 1988. 91f. Dissertação (Mestrado em 
Ciências Florestais) - Universidade Federal de Viçosa, Viçosa, 1988.

GANDOLFI, S.; LEITÃO FILHO, H. F; BEZERRA, C. L. F. Levantamento florístico e caráter sucessional das espécies arbustivo-arbóreas de uma floresta mesófila semidecídua no município de Guarulhos, SP. Revista Brasileira de Biologia, v. 55, n. 4, p. 753-767, 1995.

GOMIDE, L. R. et al. Uma nova abordagem para definição da suficiência amostral em fragmentos florestais nativos. Cerne, v. 11, n. 4, p. 376-388, 2005.

GOMIDE, L. R.; SCOLFORO, J. R. S.; OLIVEIRA, A. D. Análise da diversidade e similaridade de fragmentos florestais nativos na bacia do rio São Francisco, em Minas Gerais. Ciência Florestal, Santa Maria, v. 16, n. 2, p. 127-144, 2006.

HOWE, H. F. Seed dispersal by birds and mammals: implications for seedling demography. In: BAWA, K. S.; HADLEY, M. (Ed.) Reproductive ecology of tropical forest plants. Paris: UNESCO, 1990. p. 191-218.

KOVACH, W. L. MVSP - A MultiVariate Statistical Package for Windows, ver. 3.1. Pentraeth: Kovach Computing Services, 2005.

LEPS, J.; STURSA, J. Species-area curve, life history strategies, and succession: a field test of relationships. Vegetatio, v. 83, p. 249-257, 1989.

MACEDO, J. Os cerrados brasileiros: alternativas para a produção de alimentos no limiar do século XXI. Revista de Política Agrícola. São Paulo, v. 4, n. 2, p. 11-18, 1995.

MAGURRAN, E. A. Ecological diversity and its measurement. Princeton University Press, 1988.

MARIMON, B. S.; FELFILI, J. M.; LIMA, E. S. Floristic and phytosociology of the gallery forest of the Bacaba stream, Nova Xavantina, Mato Grosso, Brazil. Edinburch Journal of Botany, Edinburch, v. 59, n. 2, p. 303-318, 2002.

MARTINS, F. R. Estrutura de uma floresta mesófila. Campinas: UNICAMP, 1991. 246 p.

MENDONÇA, R. C. et al. Flora vascular do Cerrado. In: SANO, S. M.; ALMEIDA, S. P. (Ed.) Cerrado: ambiente e flora. Planaltina: EMBRAPACPAC, 1998. p. 89-168.

MORELLATO, L. P. C. Frutos, frugívoros e a dispersão de sementes. In: MORELLATO, L. P. C.; LEITÃO-FILHO, H. F. (Ed.). Ecologia e preservação de uma floresta tropical urbana: Reserva de Santa Genebra. Campinas: UNICAMP, 1995. p. 64-65.

MORELLATO, L. P. C.; LEITÃO-FILHO, H. F.
Padrões de frutificação e dipsersão na Serra do Japí. In: MORELLATO, L. P. C. (Ed.) História natural da serra do Japí: Ecologia e preservação de uma área florestal no sudeste do Brasil. Campinas: UNICAMP, FAPESP, 1992. p. 112-137.

MOSCOVICH, F. A.; BRENA, A. B.; LONGHI, S. J. Comparação de diferentes métodos de amostragem, de área fixa e variável, em uma floresta de Araucaria angustifolia. Ciência Florestal, Santa Maria, v. 9, n. 1, p. 173-191, 1999.

MUELLER-DOMBOIS, D.; ELLENBERG, $\mathrm{H}$. Aims and methods of vegetation ecology. New York: Willey, 1974. $574 \mathrm{p}$.

NUNES, Y. R. F. et al. Variações da fisionomia, diversidade e composição de guildas da comunidade arbórea em um fragmento de floresta semidecidual em Lavras, MG. Acta Botanica Brasilica, v. 17, n. 2, p. 213-229, 2003.

OLIVEIRA, V. A. et al. Diagnóstico agroambiental do entorno do Parque Nacional das Emas: $1^{\mathrm{a}}$. fase - pedologia, aptidão agrícola e uso das terras. Goiânia: AGÊNCIA RURAL, 2003. 227p. (Documentos, 02/AGÊNCIA RURAL).

OLIVEIRA, P. E. A. M.; MOREIRA, A. G. Anemocoria em espécies do cerrado e mata de galeria de Brasília, DF. Revista Brasileira de Botânica, v. 15, p. 163-174, 1992.

OLIVEIRA-FILHO, A. T. et al. Effects of soils and topography on the distribution of tree species in a tropical riverine forest in south-eastern Brazil. Journal of Tropical Ecology, v. 10, p. 483-508, 1995.

OLIVEIRA-FILHO, A.T. et al. Diversity and structure of the tree community of a fragment of tropical secondary forest of the Brazilian Atlantic Forest domain 15 and 40 years after logging. Revista Brasileira de Botânica, v. 27, n. 4, p. 685-701, 2004.

OLIVEIRA-FILHO, A. T. Composição florística e estrutura comunitária da floresta de galeria do Córrego da Paciência, Cuiabá (MT). Acta Botanica Brasílica, n. 3, p. 91-112, 1989.

OLIVEIRA-FILHO, A. T.; MELLO, J. M.; SCOLFORO, J. R. S. Effects of past disturbance and edges on tree community structure and dynamics within a fragment of tropical semideciduous forest in south-eastern Brazil over a five-year period (1987-1992). Plant Ecology, v. 131, p. 45-66, 1997. PINÃ-RODRIGUES, F. C. M.; AGUIAR, I. B. Maturação e dispersão de sementes. In: AGUIAR, I. B.; PINÃ-RODRIGUES, F. C. M.; FIGLIOLIA, M. B. (Ed.) Sementes florestais tropicais. Brasília: 
ABRATES, 1993. p. 215-274.

RIBEIRO, J. F.; WALTER, B. M. T. Fitofisionomias do bioma Cerrado. In: SANO, S. M.; ALMEIDA, S. P. (Ed.) Cerrado: ambiente e flora. Planaltina: EMBRAPA-CPAC, 1998. p. 89-168.

REZENDE, A. V. Importância das matas de galeria: manutenção e recuperação. In: RIBEIRO, J. F. (Ed.) Cerrado: Matas de Galeria. Planaltina: EMBRAPA-CPAC, 1998.

SAEG - Sistema para Análise Estatística e Genética (SAEG v. 5.0). Manual de uso. Viçosa: UFVFunarbe, 1997.

SANO et al. Cerrado: ecologia e flora. Brasília: Embrapa, 2008. 1279 p.

SILVA JÚNIOR, M. C. Fitossociologia e estrutura diamétrica na mata de galeria do Pitoco, na reserva ecológica do IBGE, DF. Cerne, Lavras, v. 11, p. 147-158, 2005.

SILVA JÚNIOR, M. C. Fitossociologia e estrutura diamétrica da mata de galeria do Taquara, na Reserva Biológica do IBGE, DF. Revista Árvore, v. 28, n. 3, p. 419-428, 2004.

SILVA JÚNIOR, M. C. Comparação entre matas de galeria no Distrito Federal e a efetividade do código florestal na proteção de sua diversidade arbórea. Acta Botanica Brasílica, v. 15, n. 1, p. 111-118, 2001.

SILVA JÚNIOR, M. C. et al. Análise da flora arbórea de Matas de Galeria no Distrito Federal: 21 levantamentos. In: RIBEIRO, J. F.; FONSECA, C. E. L.; SOUSA-SIlVA, J. C. Cerrado: caracterização e recuperação de Matas de Galeria. Planaltina, DF: EMBRAPA Cerrados, 2001. p. 142-191.

SILVA JÚNIOR, M. C. Comunidades de árvores e sua relação com os solos na Mata do Pitoco, Reserva
Ecológica do IBGE, Brasília-DF. Revista Árvore, v. 22, p. 29-40, 1998.

SILVA JÚNIOR, M. C. et al. Análise florística de matas de galeria no Distrito Federal. In: RIBEIRO, J. F. Cerrado - Matas de Galeria. Planaltina, DF: EMBRAPA-CPAC, 1998. p.53-84.

SILVA JÚNIOR, M. C. Tree communities of the gallery forest of the IBGE Ecological Reserve, Federal District, Brazil. 1995. 257 f. (D. Phil. Thesis) - University of Edinburgh, Edinburgh, 1995.

SOUZA, P. B. de. et al. Florística e estrutura da vegetação arbustivo-arbórea do sub-bosque de um povoamento de Eucalyptus grandis W.Hill ex Maiden em Viçosa, MG, Brasil. Revista Árvore, v. 31, p. 533-543, 2007.

TABARELLI, M. et al. Desafios e oportunidades para a conservação da biodiversidade na Mata Atlântica brasileira. Megadiversidade, v. 1, n. 1, p. 132-138, 2005.

TEIXEIRA, A. P. de; RODRIGUES, R. R. Análise florística e estrutural do componente arbustivoarbóreo de uma floresta de galeria no município de Cristais Paulistas, SP, Brasil. Acta Botânica Brasílica, v. 20, n. 4, p. 803-813, 2006.

TONIATO, M. T. Z.; OLIVEIRA-FILHO, A. T. Variations in tree community composition and structure in a fragment of tropical semideciduous forest in southeastern Brazil related to different human disturbance histories. Forest Ecology and Management, v. 198, n. 3. p. 319-339, 2004.

VALE, V. S. et al. Composição florística e estrutura do componente arbóreo em um remanescente primário de floresta estacional semidecidual em Araguari, Minas Gerais, Brasil. Hoehnea, v. 36, n. 3, p. 417-429, 2009. 